\title{
Detection of volatile signature gases for electrical fires with semiconductor gas sensors
}

\author{
Wenjia Chen, Aihua Yu, Jianxin Yi * \\ State Key Laboratory of Fire Science, Department of Safety Science and Engineering, University of \\ Science and Technology of China, Hefei, Anhui, 230026, P.R. China \\ Corresponding author's e-mail address: yix@ustc.edu.cn
}

\begin{abstract}
Electrical fires caused by over-heated PVC cables may be detected at the early stage by using proper gas detectors. Nevertheless, the composition of the gases released and thus the key components as fire signatures have not yet been determined. This work determined the compositions of the vapors emitted from several over-heated PVC cables, quantified the key components, and measured them as fire signatures with semiconductor gas sensors. Two major species ubiquitously present in the vapors of different cables were identified. These species were tested as possible fire signatures with commercial TGS chemiresistive gas sensors by using vapors of their standard chemicals. The sensor response to these species alone was very close to that to the cable vapors. Significantly enhanced response was obtained for $\mathrm{SnO}_{2}$ nanofiber gas sensors. These results demonstrate that these identified species can be considered as signature gases for electrical fires, and can be effectively detected with semiconductor gas sensors.
\end{abstract}

Key words: gas sensors; volatile gas; detection; electrical fires

\section{Introduction}

Electrical fires cause severe property damages and casualties each year in modern society. Conventional fire detectors based on the detection of smoke, temperature, or flame do not satisfy all the requirements for early, fast, and accurate detection [1]. Electrical fires are to a great extent caused by overheating and combustion of malfunctioned electrical cables and wires.

Recently, gas sensors have been found to be able to respond to the emitted vapors of overheated PVC cables, which show great promise for early detection of electrical fires [2]. However, the composition of the emitted vapors in these studies has not been undetermined, particularly for the lower temperatures before severe degradation of the PVC insulation.

In this study, compositions of vapors emitted from nine different overheated commercial PVC cables were systematically examined with TG-FTIR and GC-MS. Species suitable as the signature gases for early detection of the PVC cable fires were proposed and quantitatively determined. Detection of the signature gases and the cable vapors by commercial TGS $\mathrm{SnO}_{2}$ chemiresistor gas sensors was conducted. Results on sensor development will also be presented.

Vapor composition and signature gas

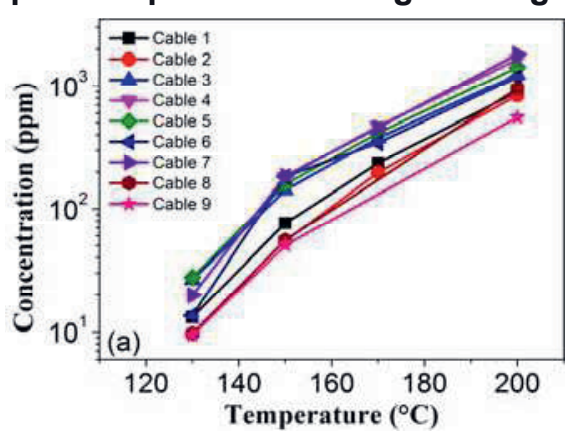

Fig.1. Calibrated concentration of one signature species at different cable temperatures.

The saturation concentrations of the proposed signature gases were determined through external standard calibration. It can be seen in Fig. 1 that for the released vapors of heated cables, the concentrations of one major species increased with increasing cable temperature.

\section{Sensing performance}

As shown in Fig. 2, the resistance of the TGS sensor decreased rapidly and substantially 
upon exposure to the two signature gases. When the signature gases were removed, the sensor resistance was recovered gradually back to the initial values.

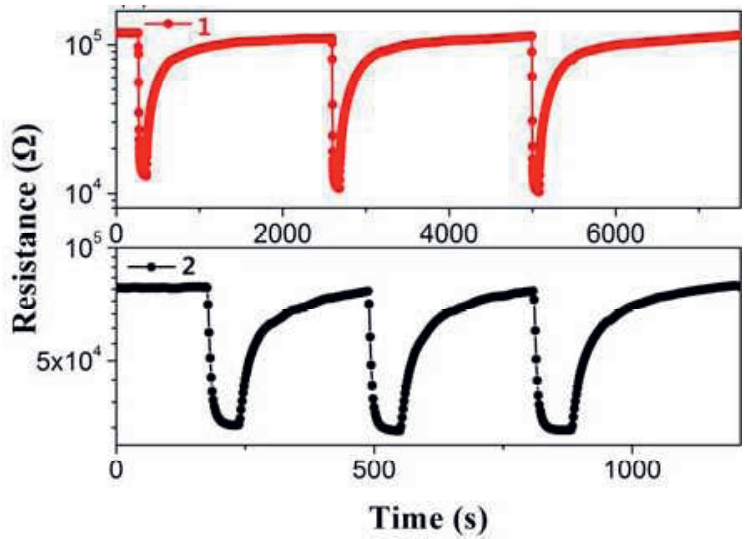

Fig.2. Resistance variation of the gas sensor to 100 ppm vapors of standard chemicals of the signature species at a sensor temperature of $190^{\circ} \mathrm{C}$.
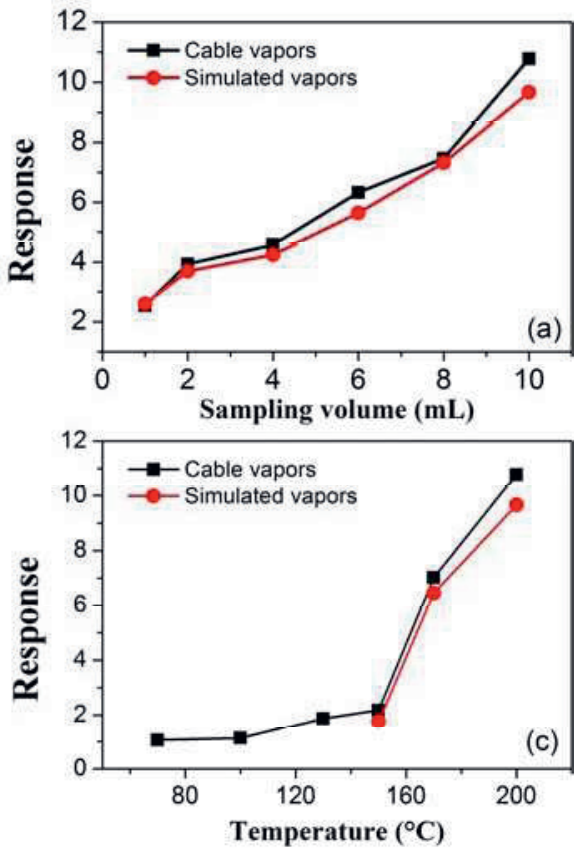

Fig.3. Variation of sensor response with (a) the sampling volume and (c) heating temperature for simulated and actual vapors for cable 1 .

Fig. 3 compares the sensor response to the cable vapors with that to the simulated vapors, the latter obtained from mixture of standard chemicals for the signature species with relevant concentrations. The gas concentration in the sensor testing chamber was adjusted by varying the sampling volume of the vapors or the heating temperature of the cables / standards. It can be seen that for the cable vapors, the sensor response increased monotonically with either the sampling volume or heating temperature, i.e. with increase of the gas concentration. Furthermore, the sensor response for the cable vapors matched very closely with that for the simulated vapors in all cases.

These results showed that the sensor response to the identified species contributed dominantly to that to the cable vapors. Therefore, these species can be used as the signature gases for early detection of electrical fires based on gas sensors.

Recent experimental results indicated that $\mathrm{SnO}_{2}$ nanofiber sensors exhibited significantly enhanced response to these signature gases in comparison to the TGS sensors.

\section{References}

[1] R. Bogue, Sensors for fire detection, Sensor Review, 33 (2013) 99-103. DOI: $10.1108 / 02602281311299635$

[2] J. Knoblauch, N. Illyaskutty, H. Kohler, Early detection of fires in electrical installations by thermally modulated $\mathrm{SnO}_{2}$ /additive-multi sensor arrays, Sensors and Actuators B-Chemical, 217 (2015) 36-40. DOI: 10.1016/j.snb.2015.02.014 